\title{
Optical recording of information pits in thin layers of chalcogenide semiconductors
}

\author{
A.N. Morozovska*, S.A. Kostyukevych**, L.L. Nikitenko, A.A. Kryuchin***, \\ A.A. Kudryavtsev, P.E. Shepeliavyi, N.L. Moskalenko \\ V. Lashkaryov Institute of Semiconductor Physics, NAS of Ukraine, 41, pr. Nauky, 03028 Kyiv, Ukraine \\ *morozo@mail.i.com.ua,**sekret@spie.org.ua. \\ *** Institute for Information Recording, NAS of Ukraine, 2, Shpak street, 03113 Kyiv, Ukraine
}

\begin{abstract}
The paper is devoted to the theoretical consideration of the question how to record pits with the necessary height profile in photosensitive materials by varying their heat conductivity, photosensitivity, optical absorption, as well as the recording gaussian beam intensity, radius and exposure time. A rather simple analytical expression for the height profile of pits has been derived. It has been shown that this profile depending on photosensitive material parameters and recording beam characteristics could be almost rectangular, flat with rounding edges, spherical or parabolic. The proposed model describes adequately the height profile of pits recorded in thin layer chalcogenide semiconductors by a gaussian laser beam.
\end{abstract}

Keywords: gaussian beam, information pit shape, chalcogenide semiconductor.

Paper received 10.02.04; accepted for publication 30.03.04.

\section{Introduction}

In recent years optical compact disks $(\mathrm{CD})$ become convenient and irreplaceable tools for information storage and distribution [1-3]. Numerous experimental and theoretical investigations devoted to the information recording and reading by optical methods has already been performed [1]. Despite a plethora of achievements, a great amount of different problems concerning information recording and reproduction remains unsolved. Let us discuss one of them.

A laser beam focused on $\mathrm{CD}$ data surface is used to read the recorded binary information. Usually the binary data are recorded in the form of pits (or bumps) covered with a very thin highly reflective layer. Light reflection from the plane surface between the pits and the surface of profiled pits ensures the readout signal.

It is well known that the strength and quality of the readout signal strongly depend on the height profile of the pit [4-5]. The height profile of information pits on the data surface of an optical disk in the most important for applications cases are rectangular or trapezoidal. As previously suggested $[6,7]$ the optimum height of the pits should be a quarter wavelength of the readout laser light. However, the authors of the publication [8] have recently shown that "a quarter wavelength deep pits are optimum for the readout signal only when a focused uniform beam illuminates rectangular pits. The optimum height of the pits increases to one-half of the wavelength or even greater value when the effect of edge rounding and sloping of side-walls of the pits is taken into account". It is obvious that the ideal rectangular pit is no more than a mathematical abstraction. Moreover, edge rounding of pits' sidewalls is the merit for $\mathrm{CD}$ manufacturing allowing for the sharp-edge damage of pits when a replica is removed from the mold [9]. Keeping in mind all the aforementioned facts, the clear and simple recommendations how to record pits with the "flat" tops, slightly rounding edges, standard proportions and sizes appeared to be rather urgent.

This paper is devoted to the theoretical consideration of the question how to record pits with the necessary profile in photosensitive materials (PSM) by varying the PSM heat conductivity, photosensitivity, absorption, as well as the recording gaussian beam intensity, radius and exposure time (see Fig.1). We used the following assumptions:

a) A thin photosensitive film is exposed by a focused gaussian laser beam, centered in the coordinate origin. 


\section{A.N. Morozovska et al.: Optical recording of information pits in thin layers ...}

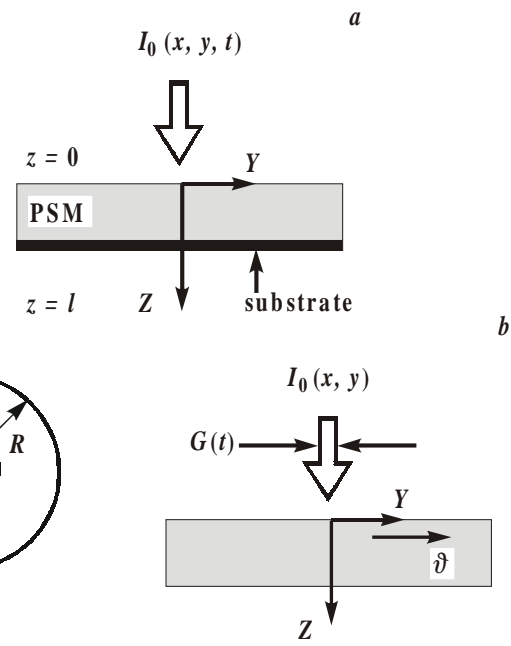

Fig. 1. The scheme of the recording process in PSM (a). The scheme of the recording on disk $(b)$.

b) The optical absorption coefficient of this thin film remains constant under the illumination process.

c) The temperature variation outside the illuminated region is negligibly small. Moreover, the temperature variation depends linearly on the laser beam intensity inside the illuminated region.

d) The change of thin film structure caused by laser beam is the main factor that determines the photo-transformed material amount.

e) The shape and sizes of photo-transformed region could be considered as the approximate ones of the recorded pits.

The approximate formula for the photo-transformed region that determines the profile parameters (width, height and length) of the pit has been obtained. It has been shown that this profile depending on PSM parameters and recording beam characteristics could be almost rectangular, flat with rounding edges or parabolic.

\section{The model}

The intensive focused laser beam illuminates the photosensitive layer (see Fig. 1a). As to the mechanism of the recording process, we adopt the following model.

1. The optical absorption of the focused gaussian laser beam [10] in the illuminated layer $0<z<\ell$ is described by the Bouguer-Lambert-Beer law:

$$
\begin{gathered}
I(x, y, z, t)=I(x, y, 0, t) \exp (-\alpha z), \\
z \geq 0, \quad 0 \leq t \leq t_{H} .
\end{gathered}
$$

Hereinafter $I(x, y, 0, t)$ is the intensity of the focused laser beam onto the surface $z=0 . \alpha$ is the light absorption coefficient that depends on the exposing beam wavelength $\lambda$, PSM temperature and exposure. In accordance with the adopted assumption $\mathrm{b}$ ) the coefficient $\alpha$ is constant at a sufficiently small recording pulse exposure time $t_{H}$.

2. The light absorption by PSM caused the transformation of the exposed region with heat producing. We assume that the distribution of PSM temperature $T_{1}(x, y, z, t)$ is determined by the light intensity $[3,11]$ :

$$
T_{1}(x, y, z, t)=T_{0}+T(x, y, z, t) .
$$

Here $T_{0}$ is the temperature of the material before illumination, and $T(x, y, z, t)$ is the temperature increase caused by light absorption. If we assume that the substrate has rather low heat conductivity, the function $T(x, y, z, t)$ is the solution of the heat problem [12]:

$\left\{\begin{array}{l}\frac{\partial}{\partial t} T(x, y, z, t)=\gamma \cdot \Delta T(x, y, z, t)+\delta \cdot I(x, y, z, t) \\ T(t=0)=0\end{array}\right.$

Here Laplace operator $\Delta=\frac{\partial^{2}}{\partial x^{2}}+\frac{\partial^{2}}{\partial y^{2}}+\frac{\partial^{2}}{\partial z^{2}}, \gamma=v / c \rho$ is the thermal diffusivity, $v$ is the thermal conductivity, $\delta \sim \alpha / \tau_{T}$ is the heat transport coefficient, $\tau_{T} \sim c \rho$ is the thermal conductivity characteristic time. The exact solution of the generalized Cauchy problem (3) could be obtained by means of the integral transformation [11] and expressed in terms of the Poisson function [12]. Surely, for thermally isolated layer $0<z<\ell$ the appropriate boundary conditions should be taken into account.

Dissipation of the thermal flux during the laser pulse time $t_{H}$ could be neglected, if the characteristic penetration depth of the thermal flux $\chi_{T}$ is higher than the film thickness $\ell$, namely

$$
\chi_{T}\left(t_{H}\right)>\ell, \chi_{T}\left(t_{H}\right)=2 \sqrt{\gamma t_{H}} .
$$

In this case, the stationary temperature distribution exists in the illuminated region, and it is proportional to the light intensity [3, 13], namely:

$$
\begin{aligned}
& T(x, y, z, t) \sim I(x, y, z, t), \\
& -\infty<x, y<\infty, \quad 0<z<\chi_{T}, \quad \tau_{T} \leq t \leq t_{H} .
\end{aligned}
$$

As it should be expected the positive variation $T(x, y, z, t)$ sharply decreases down to zero at $t>t_{H}$ or $z>\chi_{T}$.

3 . Let us consider the small volume $d V$ of PSM with the mass $\mathrm{dm}$ around the point inside the exposed region. If at the moment of time the mass of photo-transformed material is $d m_{1}$ then $M(x, y, z, t)=\lim _{d V \rightarrow 0} \frac{d m_{1}(t)}{d m}$ is the fraction of photosensitive material involved into the photo-transformation at time $t$. It is obvious, that $0<M(x, y, z, t)<1$. Note, that the increase $d M(x, y, z, t)$ during the infinitely small time $d t$ is proportional to the product of the instant exposure $d H(x, y, z, t)=I(x, y, z, t) d t$ (certainly, if the interchangeability law takes place) and the velocity of PSM photo-transformations $F$, which is proportional to the thermal diffusivity. So, $F$ depends on temperature in accordance with the Arrhenius law, i.e. $F(x, y, z, t)=$ 


\section{A.N. Morozovska et al.: Optical recording of information pits in thin layers ...}

$=F_{0} \exp \left(-E_{a} / k_{B} T_{1}(x, y, z, t)\right)[14]$. The constant $F_{0}$ depends not only on properties of recording medium but also on the recording light wavelength $\lambda$ and, sometimes, on the light intensity. $E_{a}$ is the activation energy, and $T_{1}(x, y, z, t)$ is the absolute temperature determined from (2).

The total fraction of photo-transformed material $M(x, y, z, t \rightarrow \infty) \equiv M(x, y, z)$ could be calculated using the Kolmogorov-Awrami equation [1,3], namely:

$$
\begin{aligned}
& M(x, y, z)=1-\exp \left(-\int_{0}^{t_{H}} \frac{d t}{\tau_{m}} \cdot \frac{I(x, y, z, t)}{I_{P}} \times\right. \\
& \left.\times \exp \left(-\frac{E_{a}}{k_{B}\left(T_{0}+T(x, y, z, t)\right)}\right)\right) .
\end{aligned}
$$

The characteristic constant intensity $I_{P}$ depends on the material properties, some interpretation for it will be proposed below (see (9)). The characteristic time $1 / \tau_{m} \sim F_{0}$. For the linear mechanism of recording, the fraction of transformed PSM could be estimated as

$$
\begin{aligned}
& M(x, y, z) \approx \\
& \approx \int_{0}^{t_{H}} \frac{d t}{\tau_{m}} \cdot \frac{I(x, y, z, t)}{I_{P}} \exp \left(-\frac{E_{a}}{k_{B}\left(T_{0}+T(x, y, z, t)\right)}\right) .
\end{aligned}
$$

Also (7) is valid only for sufficiently small exposure times or/and beam intensities: $t_{H}<<\tau_{m}$.

4. The photo-transformed region (PTR), which subsequently determines the pit profile, could be calculated directly from (6). The experimental possibilities of PSM developing/etching and further information registration/ reading determine the minimum value of $M_{0}$ detectable amount, namely the inequality

$$
M(x, y, z) \geq M_{0}
$$

describes the PTR. That is why, for theoretical estimations, one could regard the distribution of $M(x, y, z)$ as an actual profile of PTR.

5. Allowing for (5)-(6) one could regard that the information recording is rather effective (i.e. $M \sim 1$ ) if $T_{1}(x, y, z, t) \sim E_{a} / k_{B}$ at $\tau_{T} \leq t \leq t_{H}$. Keeping this fact in mind, it seems convenient to rewrite (5) as follows:

$$
\begin{aligned}
& T(x, y, z, t) \approx \frac{I(x, y, z, t)}{I_{P}} \frac{E_{a}}{k_{B}}, \\
& -\infty<x, y<\infty, \quad 0<z<\chi_{T}, \quad \tau_{T} \leq t \leq t_{H} .
\end{aligned}
$$

The function $I_{P}$ formally introduced in (6), (9) has the meaning of some characteristic intensity, any increase above which can provide registration of sizable amounts of photo-transformed material in our recording conditions. Sometimes, the value $I_{P}$ is called the threshold intensity, but we would like to avoid such terminology, as chalcogenide materials do not exhibit any threshold properties in the course of photo-structural transformations.

\section{Recording by the stationary gaussian laser beam}

At the beginning, let us consider the simplest case when the laser beam is immovable and has an axial-symmetrical gaussian profile. Thus, in accordance with (1), the distribution of the intensity of the gaussian laser beam with TEM00 mode [10] focused onto the surface $z=0$ inside the photosensitive layer $0<z<\ell$ acquires the form

$$
\begin{aligned}
& I(x, y, z, t)=\frac{I_{m}}{1+z^{2} / n_{0}^{2}} \times \\
& \times \exp \left[-\frac{x^{2}+y^{2}}{r_{0}^{2}(z)}-\alpha z\right] \cdot \theta\left(t_{H}-t\right), \\
& r_{0}^{2}(z)=\rho_{0}^{2}\left(1+\frac{z^{2}}{n_{0}^{2}}\right) .
\end{aligned}
$$

Hereinafter $I_{m}$ is the recording beam maximum intensity, $\alpha$ is the light absorption coefficient, $\rho_{0}$ is the recording beam characteristic radius. The diffraction broadening of the beam is described by the diffraction Fresnel number $n_{0}=2 \pi \rho_{0}^{2} / \lambda, t_{H}$ is the exposure time and $\theta(t)$ is the Heaviside step function. Also we introduce the designation $r=\sqrt{x^{2}+y^{2}}$.

The distribution (3) of temperature increase inside the photosensitive layer was calculated numerically. As it follows from our calculations, the temperature increase depends on the dimensionless exposure time $t_{H} / \tau_{T}$ and combinations $2 \alpha \sqrt{\gamma t_{H}}, 4 \gamma t_{H} / r_{0}^{2}(z), \alpha r_{0}(z)$. The evolution of the temperature variation is depicted in Fig. 2.

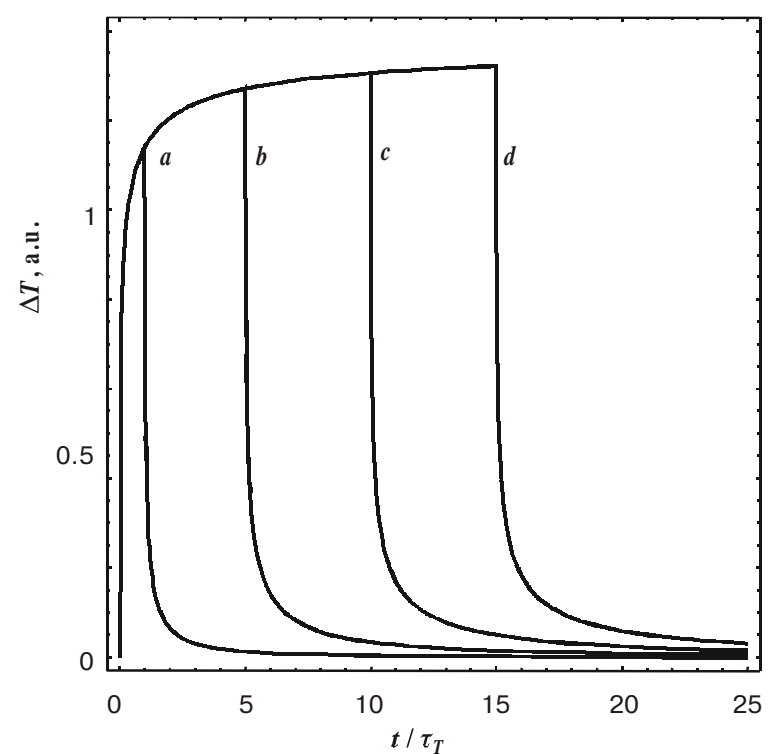

Fig. 2. The evolution of the temperature variation at different $t_{H} / \tau_{T}$ ratio: 1 (curve $a$ ), 5 (curve $b$ ), 10 (curve $c$ ), 15 (curve $d$ ). 


\section{A.N. Morozovska et al.: Optical recording of information pits in thin layers ...}

Note that (4) is valid for the reasonable values of parameters used hereinafter: on the band edge at $\lambda \sim 0.4 \mu \mathrm{m}$ and $\lambda \sim 10 \mu \mathrm{m}^{-1}[3,13]$, the recording beam characteristic radius $\rho_{0} \sim 0.5 \mu \mathrm{m}, n_{0} \sim 1 \mu \mathrm{m}, \ell \sim 0.15 \mu \mathrm{m}, \chi_{T} \sim 0.8 \mu \mathrm{m}$, $E_{a} / k_{B} T_{0} \sim 1, M_{0} \leq 0.1$. The calculations confirmed that if the inequality (4) is valid, temperature distribution similar to (9) exists. Moreover, if (4) is valid, it could be regarded as stationary at times $\tau_{T}<t<t_{H}$ and $x^{2}+y^{2} \leq r_{0}^{2}(z)$, $0<z<\min \left\{\ell, \chi_{T}\right\}$ namely:

$$
\begin{aligned}
& T(x, y, z) \approx \frac{I_{m}}{I_{P}\left(1+z^{2} / n_{0}^{2}\right)} \frac{E_{a}}{k_{B}} \times \\
& \times \exp \left(-\frac{r^{2}}{r_{0}^{2}(z)}-\alpha z\right) .
\end{aligned}
$$

Under these assumptions the fraction of transformed PSM $M(r, z)$ acquires the form:

$$
\begin{aligned}
& M(r, z) \approx 1-\exp \left(-\frac{t_{H} \cdot I_{m} / I_{P}}{\tau_{m}\left(1+z^{2} / n_{0}^{2}\right)} \times\right. \\
& \left.\times \exp \left(-\frac{r^{2}}{r_{0}^{2}(z)}-\alpha z-\frac{1}{k_{B} T_{0} / E_{a}+I(r, z) / I_{p}}\right)\right) .
\end{aligned}
$$

The fraction of transformed PSM $M(r, z)$ determined directly from (13) is depicted in Fig. 3. As it follows from (13) and Fig. 3, the approximation (7) could not be used in the case of large exposure times $t_{H} / \tau_{m} \gg 1$.

\section{Recording by the uniformly and straightforward moving gaussian laser beam}

In this section, let us consider the model situation when the axial-symmetrical gaussian laser beam moves along $\mathrm{Y}$-axis at the distance $d$ with the constant velocity $\vartheta$. In accordance with (1), the distribution of the exposing intensity and temperature inside the photosensitive layer acquires the following form

$$
I(x, y, z, t)=\frac{I_{m}}{1+z^{2} / n_{0}^{2}} \exp \left[-\frac{x^{2}+(y-\vartheta t)^{2}}{r_{0}^{2}(z)}-\alpha z\right] \times
$$

$\times \theta(d / \vartheta-t)$.

(14)

The full exposure $H(x, y, z)$ could be calculated directly from (10), namely

$$
\begin{aligned}
& H(x, y, z)= \\
& =\int_{0}^{d / \vartheta} d t \cdot \frac{I_{m}}{1+z^{2} / n_{0}^{2}} \exp \left(-\frac{x^{2}+(y-\vartheta t)^{2}}{r_{0}^{2}(z)}-\alpha z\right) .
\end{aligned}
$$

The integral in (15a) could be expressed in terms of the error function [15], i.e.:
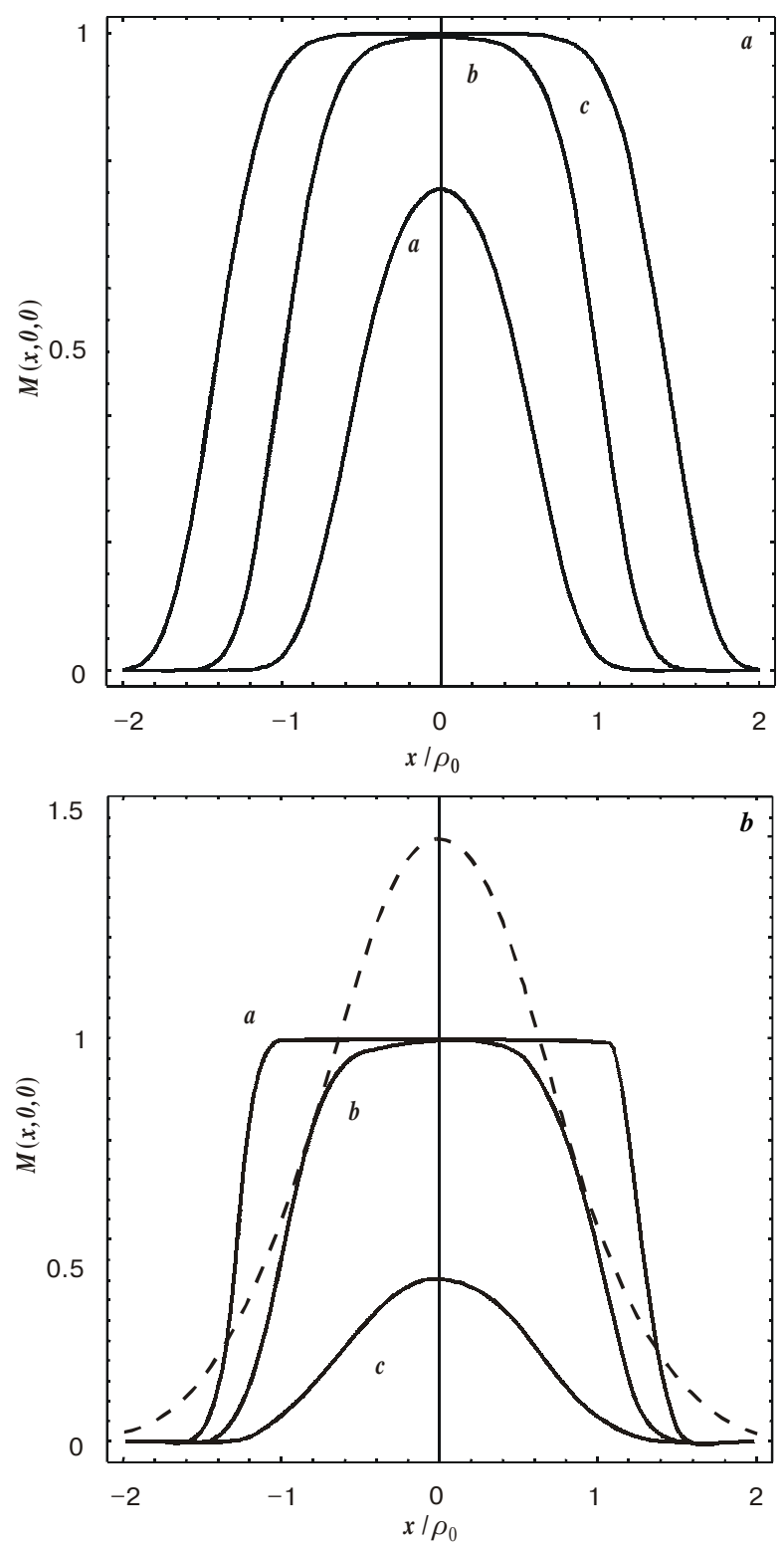

Fig. 3. The transversal cross-section of PTR $M(x, y=0, z=0)$ calculated from (13) at $t_{H} / \tau_{m}=10$ for different exposing beam intensities: $I_{m} / I_{p}=10$ (curve $\left.a\right), I_{m} / I_{p}=1.5($ curve $b), I_{m} / I_{p}=0.5$ (curve $c)(a)$; the cross-section of PTR $M(x, y=0, z=0)$ calculated from (13) at $I_{m} / I_{p}=0.5$ for different exposure times: $t_{H} / \tau_{m}=100$ (curve $a$ ), $t_{H} / \tau_{m}=10$ (curve $b$ ), $t_{H} / \tau_{m}=1$ (curve $c$ ). The dashed curve is dimensionless beam intensity $I_{b}(x, 0)=I_{m} / I_{p} \exp \left(-x^{2} / \rho_{0}^{2}\right)(b)$.

$$
\begin{aligned}
& H(x, y, z)=\frac{\sqrt{\pi} r_{0}(z)}{2 \vartheta} \frac{I_{m}}{1+z^{2} / n_{0}^{2}} \times \\
& \times\left[\operatorname{Erf}\left(\frac{d-y}{r_{0}(z)}\right)+\operatorname{Erf}\left(\frac{y}{r_{0}(z)}\right)\right] \exp \left(-\frac{x^{2}}{r_{0}^{2}(z)}-\alpha z\right) .
\end{aligned}
$$

One can obtain from (15) that the exposure spatial distribution is determined by the laser beam intensity one. The exposure value is inversely proportional to the beam 


\section{A.N. Morozovska et al.: Optical recording of information pits in thin layers ...}

velocity $\vartheta$. It is important that the profile almost coincides with the intensity profile in $X$ - and $Z$-directions. The $H(x, y, z) \quad Y$-profile flattens with increasing the ratio $d / r_{0}(z)$ (see Fig. 4). If only $d / r_{0}(z)>>1$ and $r_{0}(z)<y<d-r_{0}(z)$, one obtains that

$H(x, y, z) \approx \frac{\sqrt{\pi} r_{0}(z)}{\vartheta} \frac{I_{m}}{1+z^{2} / n_{0}^{2}} \exp \left(-\frac{x^{2}}{r_{0}^{2}(z)}-\alpha z\right)$.

Knowing the exposure (15), (16), we assume that approximate expression for temperature variation similar to (12) is valid:

$$
\begin{aligned}
& T(x, y, z, t) \approx \frac{I_{m}}{I_{P}\left(1+z^{2} / n_{0}^{2}\right)} \frac{E_{a}}{k_{B}} \times \\
& \times \exp \left(-\frac{x^{2}+(y-\vartheta t)^{2}}{r_{0}^{2}(z)}-\alpha z\right) .
\end{aligned}
$$

The maximum penetration depth of the thermal flux $\chi_{T}\left(t_{H}\right)$ is determined from (4) allowing for the exposure time $t_{H} \approx \sqrt{\pi} \rho_{0} / \vartheta$, namely $\chi_{T} \approx 2 \sqrt{\gamma \sqrt{\pi} \rho_{0} / \vartheta}$. Also in (17) we assume that (4) is valid, $\tau_{T}<t<2 d / \vartheta$, $0<z<\min \left\{\ell, \chi_{T}\right\}$ and $r_{0}(z)<y<d-r_{0}(z)$.

The fraction of photo-transformed PSM could be estimated from (6) and (17), namely one obtains that

$$
\begin{aligned}
& M(x, y, z) \approx 1-\exp \left(-\int_{0}^{d / \vartheta} \frac{d t}{\tau_{m}} \cdot \frac{I(x, y, z, t)}{I_{P}} \times\right. \\
& \left.\times \exp \left(-\frac{1}{k_{B} T_{0} / E_{a}+I(x, y, z, t) / I_{p}}\right)\right) .
\end{aligned}
$$

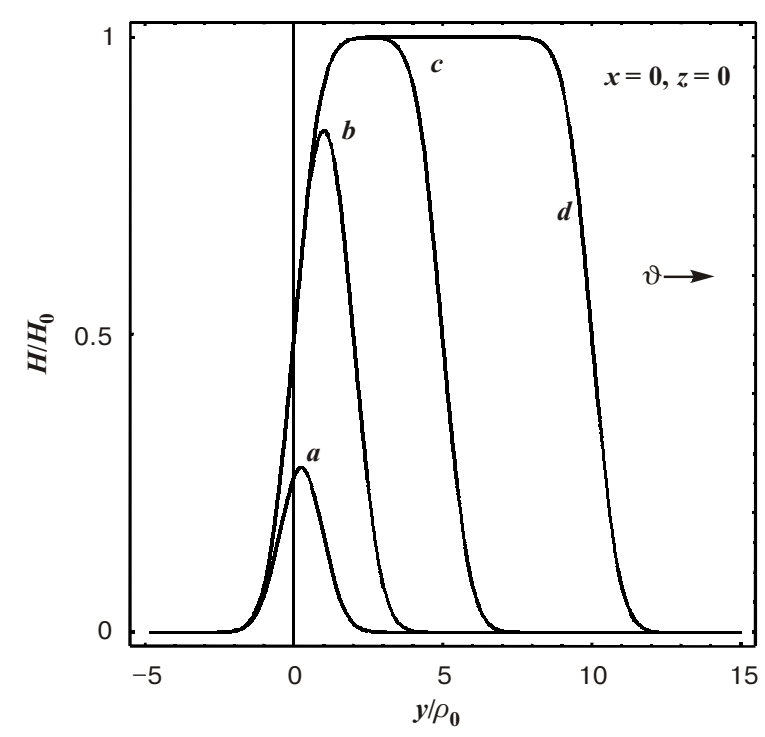

Fig. 4. The Y-profile of normalized exposure $H(x=0, y, z=0) / H_{0}$ calculated from (15) at $H_{0}=\sqrt{\pi} \rho_{0} I_{m} / \vartheta, I_{m} / I_{p}=1.5$ and different exposing distances: $d / \rho_{0}=0.5$ (curve a), $d / \rho_{0}=2$ (curve b), $d / \rho_{0}=5$ (curve c), $d / \rho_{0}=10$ (curve d).

$S Q O, 7(1), 2004$
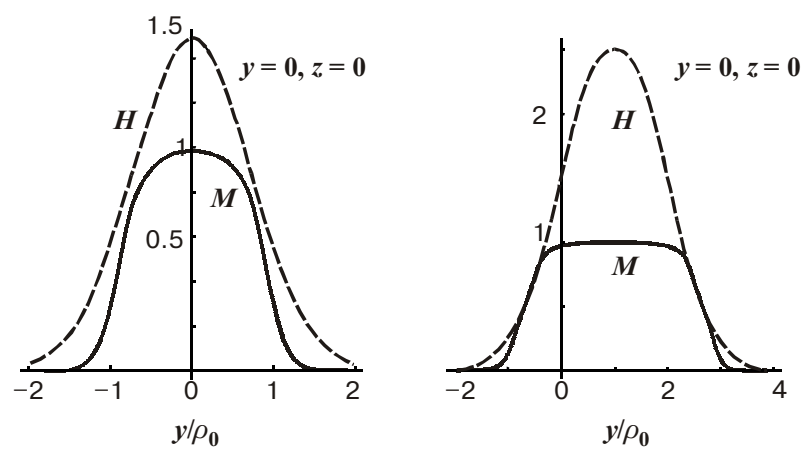

Fig. 5. The PTR $M(x, y, z)$ (curves " $M$ " calculated from (18)) and dimensionless exposure $H(x, y, z) / H_{P}$ (curves " $H$ " calculated from (15) and $\left.H_{p}=\sqrt{\pi} \rho_{0} I_{P} / \vartheta\right) X$ - and $Y$-profiles at $I_{m} / I_{p}=1.5, d / r_{0}=2$ and $r_{0} / \vartheta \tau_{m}=12.5$.

Under these assumptions, the distribution of $M(x, y, 0)$ calculated from (18) essentially differs from the distribution of dimensionless exposure $H(x, y, 0)$ calculated from (15) (see Fig. 5). It is clear from the figure that only under the condition $r_{0} / \vartheta<\tau_{m} I_{P} / I_{m}$, the formula (7) could be applied.

If only the maximum depth of thermal flux penetration is higher than the layer thickness $\left(\chi_{T} \geq \ell\right)$, the layer is rather thin $(\alpha \ell \sim 1)$ and the pulse time is much larger than the heating one $\left(2 d / \vartheta>>\tau_{T}\right)$, the isosceles trapezoid pits arise. Their shape is unambiguously determined by the gaussian recording beam radius $\rho_{0}$, the Fresnel number $n_{0}$, pit length $d$, film thickness $\ell$, absorption coefficient $\alpha$ and the depth of thermal flux penetration $\chi_{T}$. Namely the pit height $h_{p} \sim \ell$, the pit's length $b_{p} \sim d$ and the pit width determined at $z=0$ is

$a_{p} \sim 2 \rho_{0} \ln \left(\frac{I_{m}}{I_{P}}\right)$.

The tilting angle of the trapezoid pit is mainly determined by parameter $n_{0}$, absorption $\alpha$, and, therefore, it is independent over exposing beam intensity $I_{m}$.

\section{Recording by the modulated gaussian laser beam in a thin layer of chalcogenide semiconductors}

Finally, let us discuss the more realistic situation of information recording associated with the laser recording on a compact disk made of a thin layer of chalcogenide semiconductor $\mathrm{As}_{2} \mathrm{~S}_{3-\mathrm{x}} \mathrm{Se}_{\mathrm{x}}$ [16-18]. In our recent experiments [17-18] the exposure of $\mathrm{As}_{40} \mathrm{~S}_{60-\mathrm{y}} \mathrm{Se}_{\mathrm{y}}(y=0,10)$ thin layers $(100-300 \mathrm{~nm})$ has been performed by the focused laser light $(\lambda=488 \mathrm{~mm})$ with different laser beam power $(0.2-$ $0.8 \mathrm{~mW})$. Then the development of photoresist in selective etchant has been carried out. Under the positive etching, the exposed regions were completely removed, and the nonexposed ones remained on the substrate. The situation is vise versa under the negative etching. Using atomic force 


\section{A.N. Morozovska et al.: Optical recording of information pits in thin layers ...}
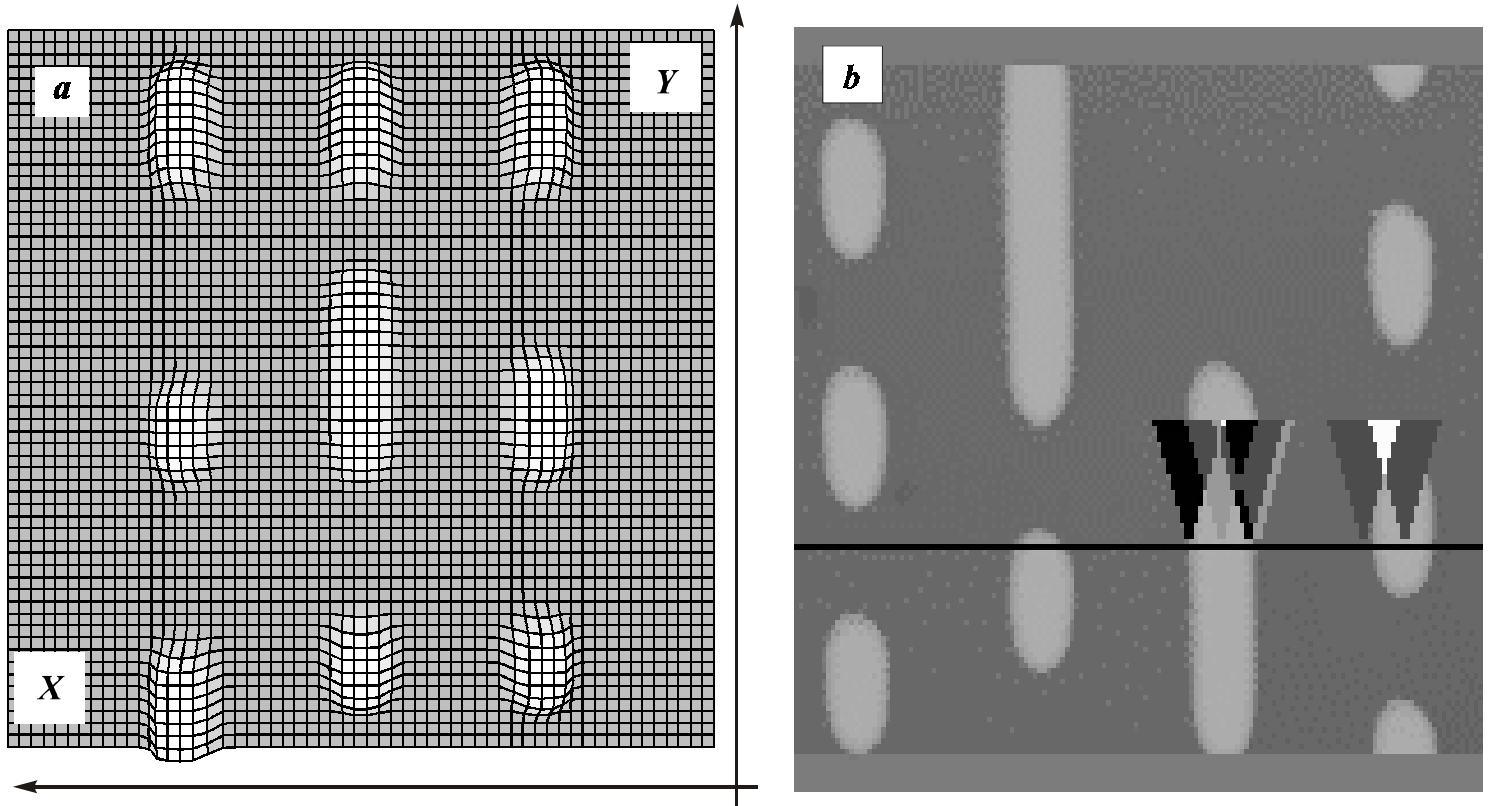

Fig. 6. The recorded pits. Top view: $a$ - theory, $b$ - experiment [16].

microscopy, we examined the obtained microstructures. The cross-section of these pits is close to the isosceles trapezoid with the tilting angle independent over exposing beam power (see comments to (19)).

For instance, let us consider that the disk with radius $R \approx 5 \mathrm{~cm}$ rotates with the constant angular velocity $\omega$ and linear velocity $\vartheta=\omega R$ (see Fig. 1b). The recording tracks occupy the area $R-L<r<R, L \approx 3 \mathrm{~cm}$. The timemodulated gaussian laser beam illuminates the track. In accordance with (1), the distribution of the exposing intensity inside the photosensitive layer acquires the following form

$$
\begin{aligned}
& I(x, y, z, t)=Q\left(t-\frac{y}{\vartheta}\right) \frac{I_{m}}{1+z^{2} / n_{0}^{2}} \times \\
& \times \exp \left[-\frac{x^{2}+(y-\vartheta t)^{2}}{r_{0}^{2}(z)}-\alpha z\right] .
\end{aligned}
$$

The modulation $Q \geq 0$ contains the reordering binary information. In order to record about $\operatorname{In} f \approx 32 \mathrm{~GB}$ on the area $S=\pi\left[R^{2}-(R-L)^{2}\right] \approx 65 \mathrm{~cm}^{2}$ one has to use the exposing spot with area not more than $s=S / \operatorname{Inf} \approx 0.2 \mu \mathrm{m}^{2}$. This requires rather small beam radii $\rho_{0} \leq 0.3 \mu \mathrm{m}$. Due to the strong inequality $\rho_{0}<<R$, the beam circular motion could be regarded as straightforward at distances $d$ approximately up to $100 \rho_{0}$. If the recording time is for $1 \mathrm{~GB}$, the disk linear velocity $\vartheta$ is about $4 \cdot 10^{3} \mathrm{~cm} / \mathrm{s}$. We would not like to discuss the reality of such recording velocities. Instead of this, we would like to stress that for rather high beam intensity $I_{m}$ even such velocities could satisfy the condition $r_{0} / \vartheta>\tau_{T}$, i.e. the basic approximate formulas for temperature redistribution (17) and the quantity of transformed matter (18) should be applied. Really, in order to estimate the $M(x, y, z)$ value from (18), it is necessary to define the exposure time as $t_{H}=\sqrt{\pi} \rho_{0} / \vartheta$.

The pits with the "flat" tops recorded by the step modulation function in accordance with (20), (18) are simulated in Fig. 6a and Fig. 7a. The electron microscopy photographs [18] of the recorded pits are represented in Figs. 6b, 7b. The cross-section view of the recorded pits is depicted in Fig. 8. It is easy to derive from the numerical data in Fig. 9 that the best coincidence between the theory and experiment [18] corresponds to the following values of the fitting parameters: $h_{p}=187.2 \mathrm{~nm}, \rho_{0}=$ $=250 \mathrm{~nm}$.

As it follows from our experiments, the pit width $a_{p}$ increases gradually with increasing the laser beam power $P$. In order to compare the formula (19) with the experimental results [17-18], we have used the following formula

$$
\begin{aligned}
& a_{p}=a_{0} \ln \left(\frac{P}{P_{0}}\right), \\
& a_{0} \approx 2 \rho_{0}, \quad P \sim I_{m}, \quad P_{0} \sim I_{P} .
\end{aligned}
$$

The dependence of the pit width ap on the laser beam power $P$ is represented in Fig. 9.

It is clear that the proposed model describes adequately the available experimental data [18]. 


\section{A.N. Morozovska et al.: Optical recording of information pits in thin layers ...}
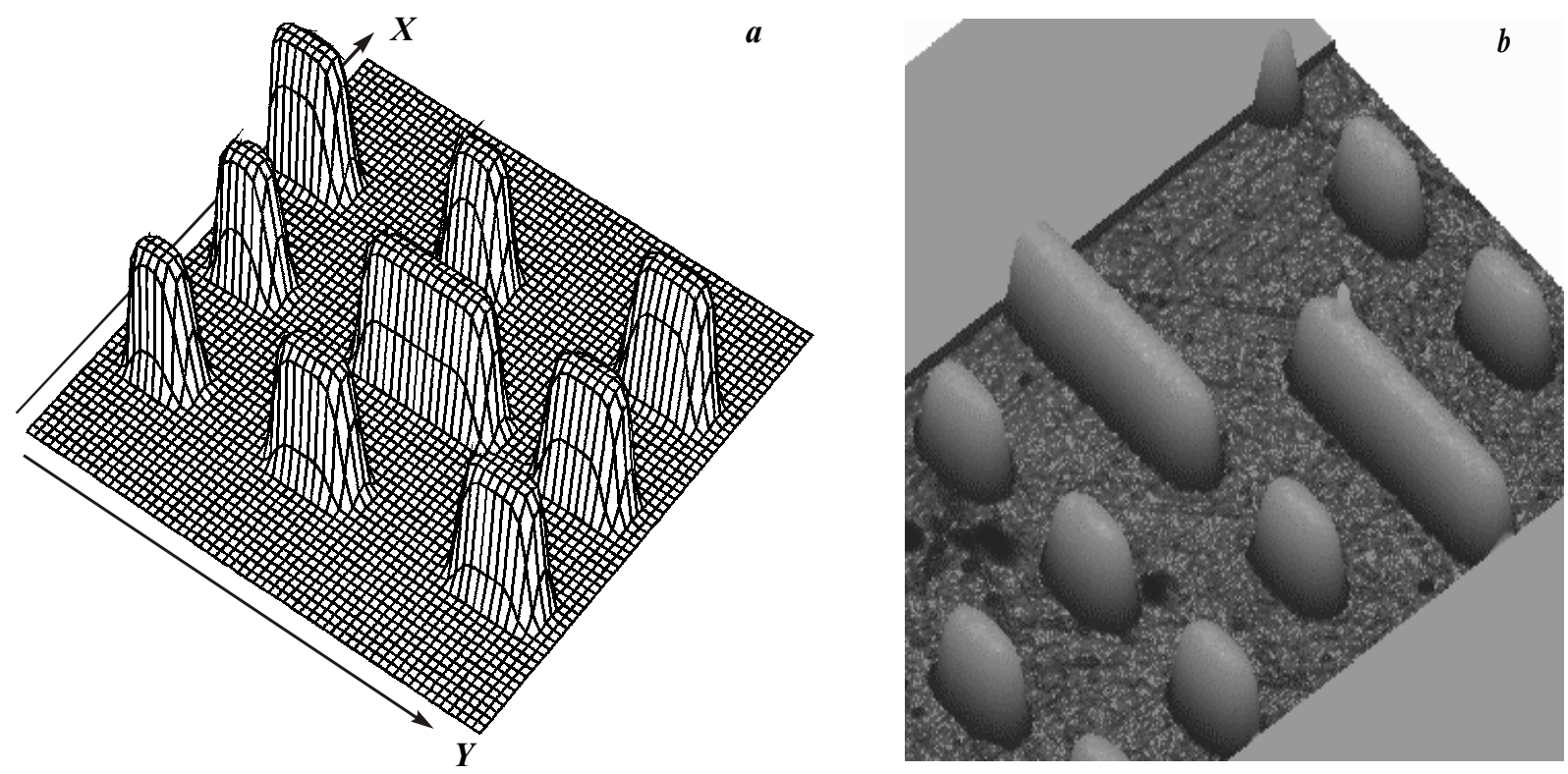

Fig. 7. The matrix with recorded pits. Side view: $a$ - theory, $b$ - experiment [16].

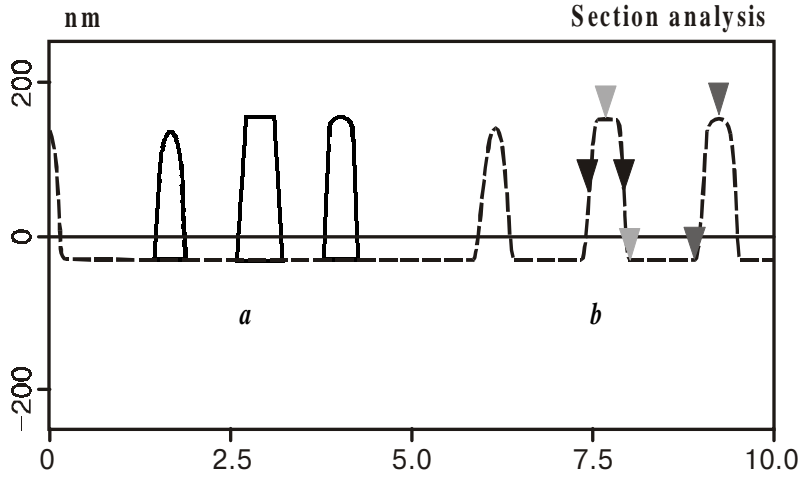

Fig. 8. The recorded pits. Cross-section view: $a$ - theory, $b-$ experiment [16].

\section{Conclusions}

- The rather simple analytical expression (18) for the pit width has been derived. It allows one to select the necessary recording conditions in order to obtain the pits with the optimum height profile [8] by using chalcogenide semiconductor thin layers and some standard previously determined conditions for etching.

- Namely, it has been shown that the height profile of the pit depending on photosensitive material parameters (heat conductivity, photosensitivity, absorption) and recording beam characteristics (gaussian beam intensity, radius and exposure time) could be almost trapezoidal $\left(\alpha \ell<1, \chi_{T}>\ell\right)$ or parabolic $\left(\alpha \ell>>1, \chi_{T}<\ell\right)$.

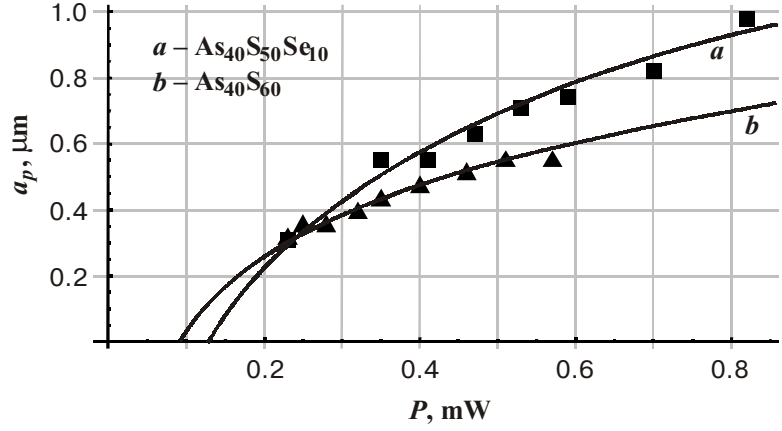

Fig. 9.The dependence of pit width $a_{p}$ over the laser beam power $P$. Solid curves - our calculations (22), squares and triangles experimental data [16]. For curves $a-a_{0}=0.5 \mu \mathrm{m}$ and $P_{0}=$ $=0.125 \mathrm{~mW}$ and curves $b-a_{0}=0.32 \mu \mathrm{m}$ and $P_{0}=0.09 \mathrm{~mW}$.

- In the most important for applications case of trapezoidal pits, their width $a_{p}$ is unambiguously determined by the gaussian recording beam maximum intensity $I_{m}$, radius $\rho_{0}$, Fresnel number $n_{0}$ and depth of the thermal flux $\chi_{T}$ correspondingly (see (19)).

- The proposed model describes adequately the height profile of the pits recorded in chalcogenide semiconductors using the Gaussian distribution of the laser beam intensity [17-18].

\section{References}

1. Marchant Alan B. Optical recording: a technical overview // Adison-Welsley Publishing Company, Inc., p. 229-255 (1990). 
2. M. Williams, A new approach to storage // TapeDisc Business, May (1997), pp. 41-43.

3. Optical disks: history state-of-art, perspectives of development / Petrov V.V., Kryuchyn A.A., Shanoylo S.M., Kostyukevich S.A., Kravets V.G., Lapchuk A.S.: Executive editor Dodonov O.G.; NAS of Ukraine. Institute for Information Recording, Institute of Semiconductor Physics. Kiev (2003), 174p.

4. H.H. Hopkins, Diffraction theory of laser readout systems for optical video disks // J. Opt. Soc. Am., 69, pp. 4-24 (1979).

5. H.H. Hopkins and C.S. Chung. Influence on the readout signal of the height profile of the pits (or bumps) on optical disks // J. Mod. Opt., 42, pp. 57-83 (1995).

6. C.M.J. Mecca, Y. Li, E. Wolf, Interference of converging spherical waves with application to the design of compact disks // Opt. Commun., 182, pp. 265-272 (2000).

7. Y. Li, C.M.J. Mecca, E. Wolf, Optimum depth of the information pits on the data surface of a compact disk// J. Mod. Opt., 50, pp. 199-206 (2003)

8. Y. Li, H. Lee, E. Wolf, Effect of edge rounding and sloping of sidewalls on the readout signal of the information pits // Opt. Eng., 42(9), pp. 2707-2720 (2003).

9. V.V. Petrov, S.M. Shanoylo, A.A. Kryuchyn et al., Optical immersion as a new way to increase information recording density // Proc. SPIE, 1731, pp. 2-12 (1991).

10. M. Born, E. Wolf, Principles of Optics, 7-th ed., Cambridge University Press. Cambridge (1999).
11. Petrov V.V., Kryuchyn A.A., Yudin G.Y. et al. The numerical simulation of crystallization process in recording media with the phase structure writing mechanism // Proc. SPIE, 2144, pp. 91-97 (1994).

12. A.N. Tikhonov, A.A. Samarskiy. The equations of the mathematical physics. Nauka, Moscow (1972).

13. J.M. Gonzalez-Leal, R. Prieto-Alcon, J.A. Angel, E. Marquez, Optical properties of thermally evaporated $\mathrm{As}_{40} \mathrm{~S}_{60-\mathrm{x}} \mathrm{Se}_{\mathrm{x}}$ films // J. Non-Crystalline Solids, 315, pp. 134-143 (2003).

14. Handbook of Photographic Science and Engineering. 768 P., Published by IS\&T, USA (1997).

15. H.B. Dwight. Tables of integrals and other mathematical data. The Macmillian company, New York (1961).

16. Indutniy I.Z., Kostyukevich S.A., Minko V.I., Stronsky A.V., Shepeliavyi P.E. Laser lithography in the layers $\mathrm{As}_{2} \mathrm{~S}_{3} / /$ Optoelectronics and Semiconductor engineering, № 25, pp. 5259 (1993).

17. Kostyukevich S.A., Indutniy I.Z., Shepeliavyi P.E. Laser recording in the layers As2S3 // Data Registration, Storage and Processing, 1(2), pp. 19-24 (1999).

18. Kostyukevich S.A., Shepeliavyi P.E., Moskalenko N.L., Vishinskaya A.V., Shanoylo S.M., Borodin Y.A., Christin V.N. The optimization of disk-originals recording with photosensitive layers As-S-Se // Data Registration, Storage and Processing, 4(2), pp. 3-10 (2002). 